\title{
Oral Supplementation of L-Arginine Improves Ventricular Remodeling by Regulating Angiogenesis and Collagen Synthesis in Myocardial Infarction Rats
}

\author{
Yonghua Liu ${ }^{1}$, Zhijuan Zhou ${ }^{2}$, Zhiling Zhu ${ }^{2}$, Wenyi Tang ${ }^{2}$, Liyun Luo ${ }^{2,}$, Chen Jian ${ }^{2}$ \\ ${ }^{1}$ Department of Interventional Medical Center, the Fifth Affiliated Hospital of Sun Yat-Sen University, Zhuhai, People's Republic of China \\ ${ }^{2}$ Department of Cardiology, the Fifth Affiliated Hospital of Sun Yat-Sen University, Zhuhai, People's Republic of China
}

Email address:

luoliyun@mail.sysu.edu.cn (Liyun Luo)

${ }^{*}$ Corresponding author

\section{To cite this article:}

Yonghua Liu, Zhijuan Zhou, Zhiling Zhu, Wenyi Tang, Liyun Luo, Chen Jian. Oral Supplementation of L-Arginine Improves Ventricular Remodeling by Regulating Angiogenesis and Collagen Synthesis in Myocardial Infarction Rats. American Journal of Clinical and Experimental Medicine. Vol. 7, No. 2, 2019, pp. 47-53. doi: 10.11648/j.ajcem.20190702.12

Received: April 8, 2019; Accepted: May 9, 2019; Published: July 9, 2019

\begin{abstract}
Objective] To investigate the effect of angiogenesis and collagen synthesis in peri-infarct area of the L-Arginine therapy for acute myocardial infarction rats. [Methods] The acute myocardial infarction rats model was established by ligation of the left anterior descending of coronary artery. Thirty male Sprague-Dawley rats were randomly divided into three groups: L-Arginine group, sham group, normal saline group (NS group). Four weeks after ligation, cardiac function, scar area, plasma concentration of BNP, angiogenesis and arteriogenesis, myocardial collagen I and eNOS protein, the mRNA expression of collagen I were studied. Echocardiography, Masson staining, enzyme-linked immunosorbent assay (ELISA), immunehistochemistry, western blot and quantitative polymerase chain (qPCR) reaction were performed. [Results] Four weeks after ligation, compared with the control group, LVEF, LVFS were higher in L-Arginine group, While LVEDD and LVESD decreased $(\mathrm{P}<0.01)$. Average scar percentage and plasma concentration of BNP were lower in L-Arginine group $(\mathrm{P}<0.01)$. The CD31-positive microvessels and $\alpha$-SMA positive microvessels in peri-infarct area were higher in L-Arginine group (P $<0.01$ ), while collagen I protein and mRNA expression was decreased in this group $(\mathrm{P}<0.01)$. [Conclusions] L-Arginine improves cardiac function and reduces infarction size in AMI rats, the possible mechanism is related to dual function of promoting angiogenesis and arteriogenesis, regulating collagen I expression is also one of the important mechanisms.
\end{abstract}

Keywords: Acute Myocardial Infarction, Angiogenesis, L-Arginine

\section{Introduction}

To reduce the mortality of patients with acute myocardial infarction (AMI) and improve their cardiac function and clinical outcomes, the use of adjuvant therapy to improve the prognosis of AMI, including stem cell transplantation and cytokine therapy, has gradually been studied by scholars [1]. Reperfusion of the occluded coronary artery is the most important way to save the ischemic myocardium. Although the reperfusion therapy can considerably reduce the mortality rate of AMI, the level of rehospitalization because of ischemic cardiomyopathy due to AMI is still high. Therapeutic angiogenesis is an attractive alternative approach to the treatment of myocardial ischemia, in which the induction of angiogenesis is used to preserve cardiac function by promoting the growth of new blood vessels and facilitating the production of new cardiomyocytes of the ischemic region [2, 3]. Among these substances that may promote blood vessel growth, L-Arginine has become a hot topic for its dual role in promoting angiogenesis and improving vascular endothelial function [4].

L-Arginine is a semi-essential amino acid that can upregulate the expression of hypoxia-inducible factors under hypoxic conditions, reduce the damage to endothelial cells under various hypoxia conditions and improve the function of vascular endothelium, also it has obvious promotion effect of angiogenesis in ischemic areas [5]. However, whether L-Arginine can improve the ventricular remodeling after 
myocardial infarction was not completely clear, this study intends to explore the mechanism of L-Arginine myocardial protection in terms of angiogenesis and its effect on collagen-I expression.

\section{Materials and Methods}

\subsection{Animals}

Male Sprague-Dawley rats (weighing 250 $\pm 50 \mathrm{~g}, 8-10$ weeks old) were obtained from the Experimental Animal Center, Sun Yat-Sen University, China. All animals received humane care in accordance with the guidelines issued by the National Science and Technology Commission of China. This experiment was approved by the institutional ethics committee. Rats were housed in groups of three per cage, fed with free access to a formula diet and clean water, at $20-24^{\circ} \mathrm{C}$.

\subsection{Ligation of the Left Coronary Artery and Treatment}

The surgery of coronary artery ligation was performed as reported earlier [6]. Rats were anesthetized using 1\% pentobarbital administered by intraperitoneal injection (30-45 $\mathrm{mg} / \mathrm{kg}$ ), under conditions of mechanical ventilation at $65-80$ breaths $/$ minute after intubation and a tidal volume of $30 \mathrm{ml} / \mathrm{kg}$. Thoracotomy was performed through the fourth/fifth intercostals space. The left anterior descending coronary artery was ligated with $6 / 0$ prolene suture, below the edge of the left atrial appendage $(2-3 \mathrm{~mm})$. Few minutes later, pallor was observed in the anterior wall corresponding to the distribution of the left anterior descending coronary artery territory. Appearance of a pale area and a huge $\mathrm{R}$ wave with a notch in electrocardiography monitoring were considered criteria for achieving of a successful model of AMI.24 hours after ligation of the coronary artery, Rats were randomized into three groups: Sham operation group, $A(n=10)$, in which the coronary artery of the animals was not ligated after thoracotomy. The experimental rats in the control group, $\mathrm{B}$ $(n=10)$ received gavage of $10 \mathrm{~mL} / \mathrm{kg} / \mathrm{d}$ normal saline for four weeks, and those of the treatment group $\mathrm{C}(\mathrm{n}=10)$ were given L-Arginine (Sigma-Aldrich LLC, St. Louis, MO, USA) by gavage once a day $(100 \mathrm{mg} / \mathrm{kg})$ for four weeks.

\subsection{Cardiac Function Examinations}

Echocardiography examinations were performed under anesthesia using $1 \%$ pentobarbital by intraperitoneal injection. Transthoracic echocardiography was performed before the surgery (baseline data) and four weeks after ligation with the Philips ultrasound system (Philips IE33, Eindhoven, the Netherlands), equipped with a $12 \mathrm{MHZ}$ linear probe. The scanning speed was $150 \mathrm{~mm} / \mathrm{s}$. The left ventricular end-diastolic dimension (LVEDD) and left ventricular end-systolic dimension (LVESD) were measured, According to the obtained data, left ventricular end-diastolic volume (LVEDV), left ventricular end-systolic volume (LVESV), left ventricular ejection fraction (LVEF), and left ventricular fraction shortening (LVFS) were calculated using the Teichholz formula [7]:

$$
\begin{gathered}
\text { LVESV }=\left(7.0 \times \text { LVESD }^{3}\right) /(2.4+\text { LVESD }) \\
\text { LVEDV }=\left(7.0 \times \text { LVEDD }^{3}\right) /(2.4+\text { LVEDD }) \\
\text { LVEF }=(\text { LVEDV-LVESV }) / \text { LVEDV } \\
\text { LVFS }=(\text { LVEDD-LVESD }) / \text { LVEDD }
\end{gathered}
$$

\subsection{Scar Area Analysis}

Four weeks after coronary ligation, the rats were anesthetized by pentobarbital, and their hearts were excised, washed with cold saline to remove the residual blood from the ventricles, and fixed in $4 \%$ formalin. After embedding in paraffin, the ventricles were sectioned into $5-\mu \mathrm{m}$ transverse slices. In order to evaluate the infarct size, Masson's trichrome staining was performed, and the stained sections were captured as images and photographed under a microscope (CCD TP510, Optec, Inc. Lowell, USA). The areas of fibrin deposition and those of viable tissue were measured, and the ratio fibrin deposition area/viable tissue area was analyzed by Image Pro-Plus software [8].

\subsection{Enzyme-linked Immunosorbent Assay (ELISA)}

Blood samples were collected from the abdominal aorta before the rats were euthanized. The blood serum was separated, and the levels of plasma concentration of brain natriuretic peptide (BNP) were measured by ELISA according to manufacturer's instructions (CBS-E07972R, CUSABIO, Wuhan, Hubei, China) [9].

\subsection{Imunohistochemical Analysis}

The rat hearts were fixed and embedded in frozen section medium. Immuno-histochemical methods were applied to evaluate neovascularization and capillary and arteriole density of the peri-infarct area. For this purpose, the following antibodies were used: anti-CD31 antibody (Abcam, Cambridge, MA, USA, catalog\#Ab5694) for identification of endothelial cells and anti- $\alpha$ smooth muscle actin antibody (Novus Biological, Littleton, CO, USA, NB100-2284) for identification of the smooth muscle cells [10]. Briefly, five fields were selected and imaged in the peri-infarct zone, and the mean total CD31-positive micro-vessels and total smooth muscle alpha actin-positive micro-vessels were counted to represent capillary density and arteriole density.

\subsection{Western Blotting}

Tissue specimens were collected from the infarct border zone, and $300 \mathrm{uL}$ of RIPA was added to the mashed tissue after centrifugation. After their homogenization for 5-8 min, the intermediate layer of liquid was collected following repeated cycles of centrifugation. Aliquots of $20 \mathrm{uL}$ of whole-cell lysates from each sample prepared in a buffer containing $2 \%$ sodium dodecyl sulfte (SDS) were separated by electrophoresis on a SDS-PAGE gel. Then they were transferred into a polyvinylidene fluoride (PVDF) membrane blot. After blocking with 5\% skim milk, the blot was incubated with primary antibodies against collagen I (Abcam, 
Cambridge, MA, USA, \#ab34710, 1:3000 dilution), at $4{ }^{\circ} \mathrm{C}$ overnight. The blot was washed twice every seven minutes using Tris-buffered saline with tween (TBST). Next, it was incubated with an HRP anti-rabbit secondary antibody (Forevergen, Guangzhou, China, \#FSA003) for 1-2 hours at room temperature. Electrogenerated chemiluminescence (ECL) was developed after another round of washing with TBST [11]. An analysis of the optical density value of the target band was conducted by Image J software.

\subsection{Quantitative Polymerase Chain Reaction, QPCR}

The collagen I target gene was searched in NCBI and its RNA was found for primer design. Then RNA extraction, reverse transcription and QPCR amplification were performed.

\section{Statistical Analysis}

All results were expressed as means \pm standard deviation (SD). The differences among different groups were evaluated for statistical significance using one-way analysis of variance (ANOVA) (normal distribution data), or Kruskal-Wallis test (non-normal distribution data), A probability value, $P<0.05$, was considered statistically significant. Statistical analyses were performed using IBM SPSS Statistics 21.0 software.

\section{Results}

\subsection{Echocardiography Findings}

Echocardiography was performed before the surgery and four weeks after ligation. Before the surgery, there were no differences among the three groups in the values of LVEDD, LVESD, and LVEF, $(P>0.05)$. As established through ANOVA, there were significant differences among these three groups in the levels of LVEDD, LVESD, LVEF, and LVFS four weeks after ligation. At 4 weeks after coronary artery ligation, echocardiography revealed that the anterior wall motion of the ventricle in the myocardial infarction group (control group) was weakened or disappeared. LVEF and LVFS, which were indicators of ventricular systolic function, were significantly lower than those in the sham operation group, and LVEDD and LVESD were significant increased $(P$ $<0.01)$. Compared with the control group, LVEF and LVFS in the L-Arginine treatment group were significantly higher, while LVEDD and LVESD were significantly lower $(P<$ 0.01). See Table 1 and Figure 1.

Table 1. Echocardiography Findings of three groups (mean \pm SD).

\begin{tabular}{llll}
\hline Groups & LVEDD (mm) & LVESD (mm) & LVEF (\%) \\
\hline Sham & $6.61 \pm 0.86$ & $3.94 \pm 0.20$ & $79.06 \pm 2.89$ \\
Control & $9.06 \pm 0.23^{*}$ & $7.32 \pm 0.30^{*}$ & $35.20 \pm 0.91^{*}$ \\
L-Arg & $7.82 \pm 0.36^{*}$ & $6.29 \pm 0.17^{*}$ & $48.20 \pm 1.06^{*}$ \\
\hline
\end{tabular}

*ANOVA and Post-hoc comparisons, $P<0.01$.
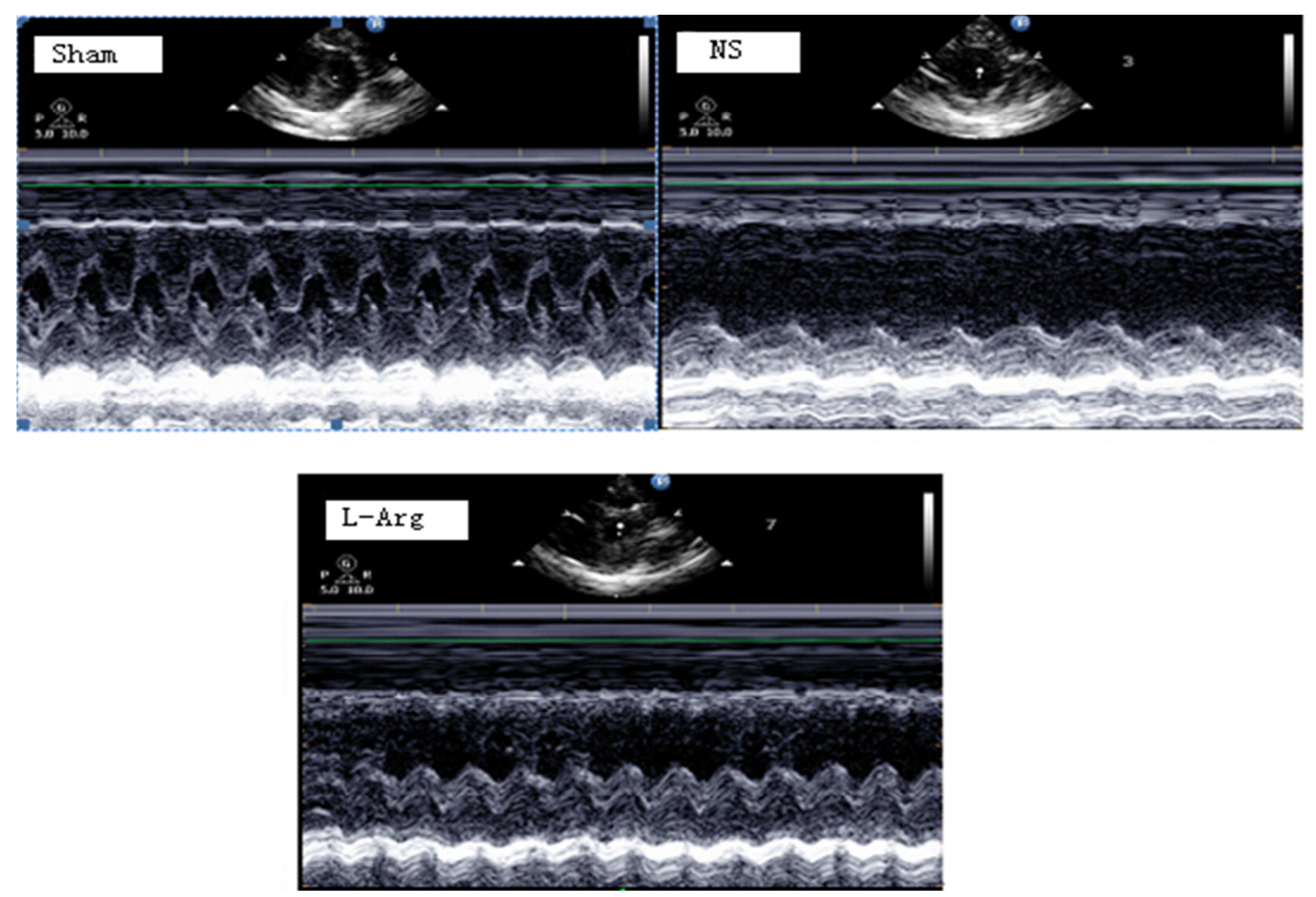

Figure 1. Echocardiography Findings in three groups. 


\subsection{Scar Area Measurements}

The Masson trichrome staining results are presented in Figure 2. Viable myocardium areas were stained red, whereas those with fibrosis were blue. No area was stained blue in the sham operation group. 4 weeks after coronary artery ligation, compared with the control group, the infarct size in the L-Arg group was significantly reduced $(P<0.01$, Table 2$)$.
Table 2. Scar area of three groups (mean $\pm S D$ ).

\begin{tabular}{ll}
\hline Groups & Scar area (\%) \\
\hline Sham 0 & \\
NS & $35.02 \pm 2.40$ \\
L-Arg & $25.11 \pm 2.17^{*}$ \\
\hline
\end{tabular}

*t-Test, $P<0.01$.
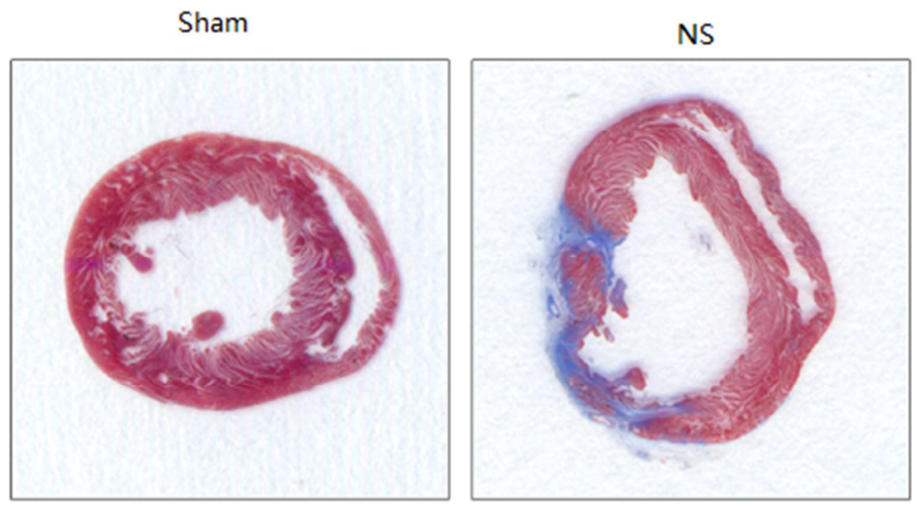

Figure 2. Masson Trichrome staining.

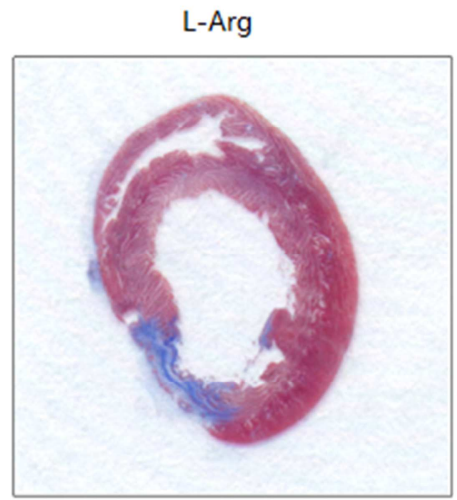

determination of CD31 in the endothelial cells of the peri-infarct area of rats in each group was shown in Fig. 3. ANOVA manifested that there were differences in capillary density (CD31 positive cells $/ \mathrm{mm} 2)$ between groups $(P<0.05)$, with the highest in sham-operated group $\left(169.2 \pm 5.78 / \mathrm{mm}^{2}\right)$. Post-hoc test showed that capillary density in L-Arginine treatment group $\left(101.4 \pm 6.74 / \mathrm{mm}^{2}\right)$ was higher than that in control group $\left(34.8 \pm 5.15 / \mathrm{mm}^{2}\right)(P<0.01)$, table 4 .

The number of SMA positive cells was also significantly different among the groups (Fig. 4). Sham operation $\left(42 \pm 4.56 / \mathrm{mm}^{2}\right)$ was higher than the other two groups. The arterial density (SMA positive cells $\left./ \mathrm{mm}^{2}\right)\left(20.2 \pm 3.12 / \mathrm{mm}^{2}\right)$ in L-Arginine treatment group was significantly higher than that in control group $\left(7.2 \pm 1.72 / \mathrm{mm}^{2}\right)(P<0.01)$, Table 5 .

Table 4. CD31 positive cells/ $\mathrm{mm}^{2}$ (mean $\left.\pm S D\right)$.

\begin{tabular}{ll}
\hline Groups & CD31 positive cells $/ \mathbf{m m}^{2}$ \\
\hline Sham & $169.2 \pm 5.78$ \\
NS & $34.8 \pm 5.15$ \\
L-Arg & $101.4 \pm 6.74$ \\
\hline
\end{tabular}

*ANOVA and Post-hoc comparisons, $P<0.01$.

In order to clarify the mechanism of the potential beneficial effects of L-Arginine in post-infarct pathology, angiogenesis and arteriogenesis in peri-infarct myocardium were analyzed by immunohistochemical method. Immunohistochemical
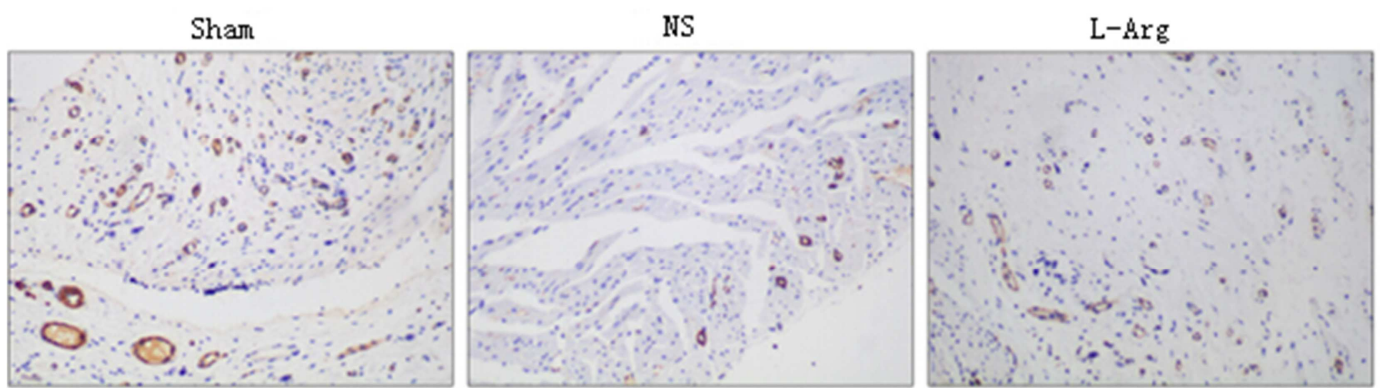

Figure 3. Immunohistochemical staining of CD31 (200x). 

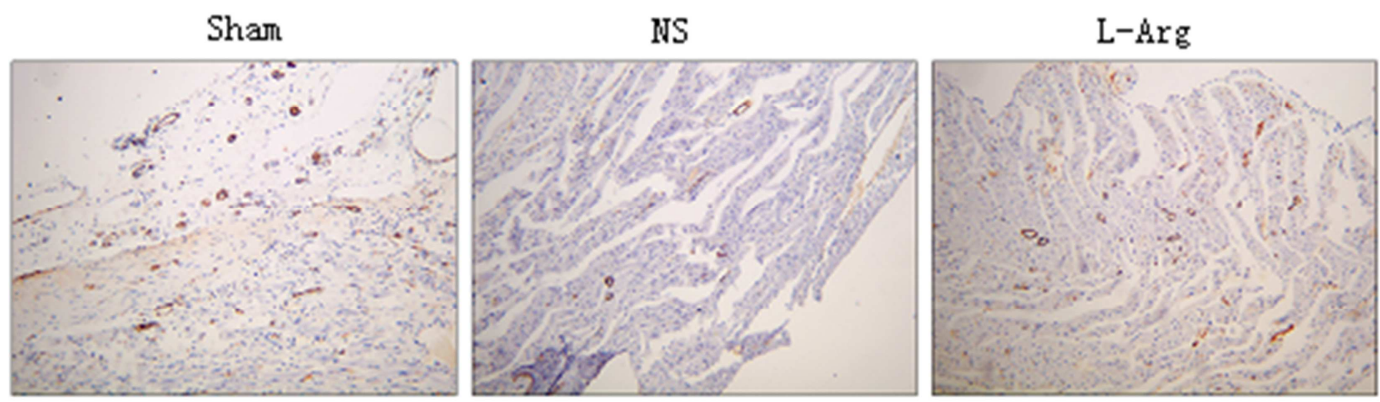

Figure 4. Immunohistochemical staining of $\alpha-S M A(100 x)$.

Table 5. Comparison of the arterial density (mean $\pm S D)$.

\begin{tabular}{ll}
\hline Groups & $\alpha$-SMA positive $/ \mathbf{m m}^{2}$ \\
\hline Sham & $42.0 \pm 4.56$ \\
NS & $7.2 \pm 1.72$ \\
L-Arg & $20.2 \pm 3.12^{*}$ \\
\hline
\end{tabular}

*ANOVA and Post-hoc comparisons, $P<0.01$.

\subsection{Western Blot Analysis eNOS and Collagen I Protein}

Four weeks after myocardial infarction, Western blot showed that the expression level of eNOS in saline control group was the lowest, while the expression level of eNOS in L-Arginine group was significantly higher than that in control group. The content of Collagen I protein in sham operation group was the lowest, compared with sham operation group, the content of Collagen I protein in L-Arginine group was lower $(P<0.01)$, The results of the densitometry analysis of protein levels in the peri-infarct area are displayed in Figure 5.

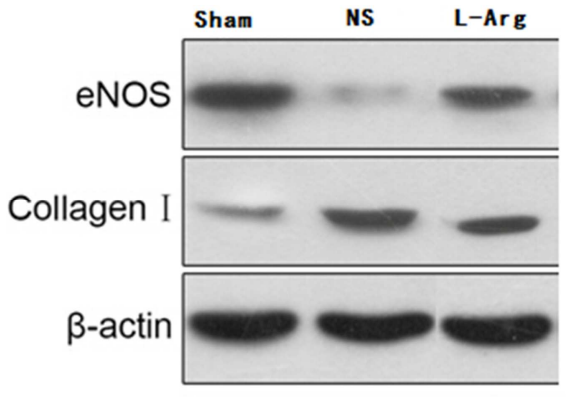

Figure 5. Western blot of eNOS and Collagen I protein.

\subsection{QPCR of Collagen I mRNA}

The results showed that the relative expression of collagen type I mRNA was different between three groups, Compared with NS group, the relative expression of collagen type I in L-Arg group was lower $(P<0.01)$, Table 6 .

Table.6. QPCR of Collagen-I $m R N A$ in peri-infarct area $(\bar{x} \pm s)$.

\begin{tabular}{lll}
\hline & n & Relative Collagen 1 mRNA level \\
\hline Sham & 10 & $0.55 \pm 0.39$ \\
NS & 10 & $3.58 \pm 2.01$ \\
L-Arg & 10 & $2.32 \pm 0.39$ \\
\hline
\end{tabular}

\section{Discussion}

Acute myocardial infarction (AMI) is still one of the diseases that seriously threaten human life. Its incidence is increasing year by year in China. At present, mature reperfusion therapy, especially percutaneous coronary intervention (PCI), can quickly access the related artery of AMI patients and restore the blood supply of myocardium as quickly as possible [12]. But some patients did not receive reperfusion therapy in time because of limited conditions. Reperfusion injury and no-reflow during operation resulted in unsatisfactory perfusion of myocardial microcirculation tissue. These patients often have obvious ventricular remodeling and eventually lead to heart failure.

The effects of L-Arginine on angiogenesis and myocardial protection in rats with acute myocardial infarction were observed in this study. The results showed that L-Arginine intervention could improve left ventricular ejection fraction, reduce infarct size, increase capillary and arteriolar regeneration in rats with acute myocardial infarction. It was also observed that plasma BNP level in L-Arginine intervention group decreased significantly, which is a cardiac insufficiency specific biomarkers for poor prognosis and severity. In this study, we found that eNOS expression in myocardium around myocardial infarction decreased significantly 4 weeks after myocardial infarction, and L-Arginine intervention could enhance eNOS expression. Therefore, we speculate that the promotion of angiogenesis by L-Arginine is mainly related to its effect on eNOS expression. The effect of L-Arginine intervention on Collagen- I, a key component of myocardial interstitial remodeling after myocardial infarction, was also preliminarily explored. The results showed that L-Arginine could reduce the content of Collagen- I in myocardium around chronic myocardial infarction.

The mechanism of the effects of L-Arginine on angiogenesis and myocardial protection has not been fully clarified. Animal experiments showed that the expression of endothelial nitric oxide synthase (eNOS) was significantly decreased in the early stage of myocardial infarction in rats [13]. The mechanism may be related to the increase of asymmetric dimethylarginine (ADMA) concentration in plasma and left ventricular infarction and marginal zone [14]. ADMA may inhibit the activity of eNOS, while eNOS can catalyze the conversion of arginine. Citrulline and nitric oxide (NO), which are powerful endogenous vasodilator factors, can inhibit leukocyte adhesion and platelet aggregation, maintain the stability of endothelial cell function. If NO synthesis is insufficient, it will affect the level of myocardial perfusion and 
angiogenesis [15]. L-Arginine is a semi-essential amino acid and a substrate of nitric oxide synthase. It can up-regulate the expression of hypoxia-inducible factors under hypoxic conditions and reduce the damage of various factors to endothelial cells under hypoxic conditions [16]. In addition, L-Arginine can antagonize the inhibition of ADMA on endothelial cell proliferation. Although it can not decrease the level of ADMA in vivo, it can restore the L-Arginine/ADMA ratio, thus antagonizing the effect of ADMA and increasing the expression and activity of eNOS mRNA. Studies have shown that eNOS plays an important role in angiogenesis and myocardial remodeling after AMI. Myocardial angiogenesis in ischemic area of eNOS knockout rats is significantly inhibited. The response to statins and the mobilization ability of endothelial progenitor cells (EPCs) are decreased. After intervention with AVE9488 (an eNOS agonist), the concentration of eNOS and EPCs increased, the neovascu-larization increased and the infarct size decreased [17, 18].

This study found that the protective effect of PIGF on myocardium may also be related to its effect on the expression of Collagen-1 protein in myocardium around infarction. Collagen- I is the main component of intercellular matrix and plays an important role in the process of myocardial fibrosis after myocardial infarction [19]. Regulation of collagen synthesis has become an important target for the treatment of ventricular remodeling. The possible mechanisms of L-Arginine reducing the expression of Collagen- I in myocardium around chronic myocardial infarction include: with the increase of neovascularization, the level of myocardial ischemia and hypoxia is improved, the expression of tissue inhibitor of matrix metalloproteinase-2 (TIMP-2) and matrix metalloproteinase-2 (MMP-2) is increased, leading to the increase of Collagen- I degradation [20].

The discovery of angiogenesis is a great progress in the treatment of ischemic heart disease. However, the understanding of its mechanism is still in its infancy. The methods of promoting angiogenesis include cytokine therapy, gene therapy and stem cell therapy. Combined therapy should be the focus and direction of future research, including combination of cytokine therapy, combined cytokine therapy and stem cell therapy. However, many cytokines promoting angiogenesis have achieved good therapeutic effects in animal experiments, but they have not achieved the expected benefits in human clinical trials. More and more studies have shown that the above-mentioned situation is related to many factors, such as patient selection, timing of application, maturity of new blood vessels, the route of administration, drug carriers, dosage and so on. The problem remains unsolved, and further studies on the mechanism and safety of large-scale clinical application need to be explored [21].

\section{Conclusion}

L-Arginine improves cardiac function and reduces infarction size in AMI rats, the possible mechanism is related to dual function of promoting angiogenesis and arteriogenesis, regulating collagen I expression is also one of the important mechanisms.

\section{Conflict of Interest Statement}

All the authors do not have any possible conflicts of interest.

\section{References}

[1] Singh P, Sethi N, Kaur N, et al. Revascularization in Severe Left Ventricular Dysfunction: Does Myocardial Viability Even Matter [J]? Clin Med Insights Cardiol, 2015, 9 (Suppl 1): 105-9.

[2] Lassaletta, A. D. Chu, L. M. Sellke, F. W. Therapeutic neovascularization for coronary disease: current state and future prospects [J]. Basic Res Cardiol, 2011, 106 (6): 897-909.

[3] Takeda $\mathrm{Y}$, Uemura $\mathrm{S}$, Iwama $\mathrm{H}$, et al. Treatment with recombinant placental growth factor (PIGF) enhances both angiogenesis and arteriogenesis and improves survival after myocardial infarction [J]. Circ J, 2009, 73 (9): 1674-82.

[4] Voisine P, Bianchi C, Khan TA, et al. Normalization of coronary microvascular reactivity and improvement in myocardial perfusion by surgical vascular endothelial growth factor combined with oral supplementation of L-Arginine in a porcine model of endothelial dysfunction. J Thoral cardiocasc Surg, 2005, 129 (6): 1414-20.

[5] He Jin, Xie Xiumei, Fangye Qing, et al. L-Arginine inhibits the proliferation of endothelial progenitor cells induced by asymmetric dimethylarginine. Chinese Journal of Arteriosclerosis, 2007, 15 (10): 751-754.

[6] J Chen, S Zheng, H Huang, et al. Mesenchymal Stem Cells Enhanced Cardiac Nerve Sprouting via Nerve Growth Factor in a Rat Model of Myocardial Infarction [J]. Current Pharmaceutical Design, 2014, 20 (12): 2023-2029.

[7] Wykrzykowska JJ, Rosinberg A, Lee SU, et al. Autologous cardiomyotissue implantation promotes myocardial regeneration, decreases infarct size, and improves left ventricular function [J]. Circulation, 2011, 123 (1): 62-9.

[8] Chen G, Nayan M, Duong M, et al. Marrow stromal cells for cell-based therapy: the role of antiinflammatory cytokines in cellular cardiomyoplasty [J]. Ann Thorac Surg, 2010, 90 (1): 190-7.

[9] Li J, Yin FF, Hou YL. Early diagnosis of rats with acute myocardial infarction by measurement of brain natriuretic peptide [J]. Exp Ther Med, 2013, 5 (4): 1201- 1205.

[10] Rendell MS, Finnegan MF, Pisarri T, et al. et al. A comparison of the cutaneous microvascular properties of the spontaneously hypertensive rat and the Wistar-Kyoto rat [J]. Comp Biochem Physiol A Mol Integr Physiol, 1999, 122 (4): 399-406.

[11] Voisine P, Bianchi C, Khan TA, et al. Normalization of coronary microvascular reactivity and improvement in myocardial perfusion by surgical vascular endothelial growth factor combined with oral supplementation of L-arginine in a porcine model of endothelial dysfunction [J]. J Thoral cardiocasc Surg, 2005, 129 (6): 1414-20. 
[12] Society of Cardiology, Chinese Medical Association. Guidelines for diagnosis and treatment of acute ST-segment elevation myocardial infarction in 2015. Chinese Journal of Cardiovascular Disease, 2015, 5: 380-393.

[13] Lu Dongfeng, Longguanzhou, Shi Shumei. Decreased expression of endothelial nitric oxide synthase gene and increased expression of endothelial nitric oxide synthase gene by L-Arginine in rats with acute myocardial infarction. Chinese Journal of Cardiovascular Disease, 2004, 10 (32): 713.

[14] Gray GA, Patrizio M, Sherry L, et al. Immunolocalisation and activity of DDAH I and II in the heart and modification post-myocardial infarction. Acta Histochem, 2010, 112 (5): 413-23.

[15] Theilmeier G, Chan JR, Zalpour S, et al. Adhesiveness of Mononuclear Cells in Hyperchole sterolemic Humans is normalized by Dietary L-Arginine. Arteriosclero Thromb Vasc Biol, 1997, 17: 3557-3564.

[16] Martínez-Romero R, Cañuelo A, Siles E, et al. Nitric oxide modulates hypoxia-inducible factor-1 and poly (ADP-ribose) polymerase-1 cross talk in response to hypobaric hypoxia. $\mathrm{J}$ Appl Physiol, 2012, 112 (5): 816-23.
[17] Fraccarollo D, Widder JD, Galuppo P, et al. Improvement in left ventricular remodeling by the endothelial nitric oxide synthase enhancer AVE9488 after experimental myocardial infarction. Circulation, 2008, 118: 818-827.

[18] Landmesser U, Engberding N, Bahlmann FH, et al. Statin-induced improvement of endothelial progenitor cell mobilization, myocardial neovascularization, left ventricular function, and survival after experimental myocardial infarction requires endothelial nitric oxide synthase. Circulation, 2004, 110: 1933-1939.

[19] Bornstein P. Matricellular proteins: an overview [J]. J Cell Commun Signal, 2009, 3 (3-4): 163-5.

[20] Lu, Z, X. Mao, L. L. Lian, F. et al. Cardioprotective activity of placental growth factor in a rat model of acute myocardial infarction: nanoparticle-based delivery versus direct myocardial injection [J]. BMC Cardiovasc Disord, 2014, 17; 14: 53.

[21] Antonio D, Lassaletta Louis M, Chu Frank W, et al. Therapeutic neovascularization for coronary disease: current state and future prospects [J]. Basic Res Cardiol, 2011, 106 (6): 897-909. 